\title{
Dual Axis Solar Tracking System for Solar Panel
}

\author{
Purnima Singh ${ }^{*}$ Roop Pahuja, Meghavi Karwasra, Sunita Beniwal, Meenakshi Bansal, \\ Anamika Dadhich \\ Dr. B.R. Ambedkar National Institute of Technology Jalandhar (Punjab), 144 011, India \\ ${ }^{*}$ Corresponding author, e-mail: purnima814@gmail.com
}

\begin{abstract}
Sustainable energy systems are necessary for the economic growth and a healthy environment. India is currently suffering from an economic slump and acute fuel shortage. To overcome these issues the use of renewable energy resources needs to be enhanced manifold. The main purpose of this paper is to present a tracking system which will cause better alignment of solar panel with sunlight and to harvest solar power. The proposed system changes its direction in two axis to trace the coordinate of sunlight by detecting change in light intensity through light dependent resistors. Hardware testing of the proposed system is done for checking the system ability to track and follow the sunlight in an efficient way. Dual axis solar tracking system superiority over stationary solar panel is also presented.
\end{abstract}

Keywords: LDR sensing arrangement, dual axis tracking, L293D, microcontroller

\section{Introduction}

The world population is increasing day by day and the demand for energy is increasing accordingly. Oil and coal, the main source of energy nowadays, are expected to end up from the world during the recent century which explores a serious problem in providing the humanity with an affordable and reliable source of energy. The need of the hour is renewable energy resources with cheap running costs. Solar energy has become a preferred because of its ubiquity, abundance and sustainability, regardless the intermittency of sunlight, solar energy is widely available and completely free of cost. It is harnessed (converted to other forms of energy) by using techniques such as solar heating photovoltaic cell, solar thermal energy, solar architecture and artificial photosynthesis. Photovoltaic is solid-state device that simply makes electricity out of sunlight, silently and with little to no maintenance, no pollution and no significant depletion of material resources [1]. However, it is costly to install but in a long term it can save more energy and offers more reduction in cost. Solar panels, also called photovoltaic or PV module as it directly converts sunlight into electricity. A photovoltaic module is an interconnected collection of cells combined into one item. When a number of solar or photovoltaic modules are installed together, this is commonly referred to as a solar array. Many schemes have been developed to incorporate the same. The most widely renowned till date are:

a. Static Solar Panel Systems

b. Time based Moving Solar Panel Systems

c. Maximum Power Point Tracking Solar Panel Systems.

Detailed analysis and work done in above mentioned schemes is discussed under paragraph of related work. Solar tracker is an electro-mechanical device for orienting a solar photovoltaic panel toward the sun to get maximum intensity of light and to keep the position of panel perpendicular to the light source. Solar powered equipment works best when pointed at or near the sun, so a solar tracker can increase the effectiveness of such equipment. The tracker will enable the panel to follow the path of the sun and produce more power as it absorbs more sunlight. In single axis solar tracking, tracker can only capture the minimum power tracking sunlight in one direction which is the elevation movements from east to west by rotating the structure along the vertical axis. Dual axis solar tracker can capture the maximum sunlight by rotating panel in two movements that is elevation and azimuth [2]. Uses of Single Axis throughout the year do not maintain the output power. The position of sun will change from the position of installed solar tracker and make the panel no more perpendicular to the sun which affects the output power. Therefore, dual-axis solar tracking is used to align solar panel perpendicular to the sun throughout the day [3]. This paper is written with the aim of designing 
efficient and cost effective tracking system by overcoming the drawbacks of the existing solar panel systems. It shows the development of a sensor based dual axis tracking system so that maximum intensity of sunlight falls on it. By using microcontroller to control the rotation of two DC motor depending on intensity of sunlight sensed by LDR arrangement.

\subsection{Related Work}

There are number of ways in which solar energy is harvested using solar panel [4]. These are stated as below: et al. Binoy Seal, 2004 [5] in his work stated one of the method is using Single axis solar tracker device, on the basis of LDR sensor values, orients the solar panel in accordance with the position of the sun. This system tracks the sun only from east to west direction. It's efficiency is less compare to dual axis solar tracker. Other than single axis sensor based tracking mechanism there are two more principles used for tracking purpose. One of which is Time based tracking mechanism by et al. K. Anusha, 2013 [6]. In this paper refers to the design of a single axis solar tracker system that automatically adjusts the optimum PV panel position with respect to the sun by means of a DC motor controlled by an intelligent controller unit that equipped with a positioning algorithm to mathematically solve the optimum tracker position for any time of the day using RTC. A practical measurement of the sun position with respect to the natural time relational table is implemented as an algorithm to track the sun position to achieve maximum energy. Finally, a comparison between the tracking system and the fixed system is made. From the experimental results, the proposed tracking system is verified more efficiently in generating energy than the fixed system. Another principle includes maximum power point tracking algorithm. MPPT or Maximum Power Point Tracking is algorithm that included in charge controllers used for extracting maximum available power from PV module under certain conditions. The voltage at which PV module can produce maximum power is called 'maximum power point' (or peak power voltage). Maximum power varies with solar radiation, ambient temperature [6].The major principle of MPPT is to extract the maximum available power from PV module by making them operate at the most efficient voltage (maximum power point). That is to say: MPPT checks output of PV module, compares it to battery voltage then fixes what is the best power that PV module can produce to charge the battery and converts it to the best voltage to get maximum current into battery [7]. It can also supply power to a DC load, which is connected directly to the battery.

\section{System Design}

This section explains the workflow of modeling and designing of the prototype hardware and also explains the tracking software algorithm.

\subsection{Proposed Work}

Existing Methods for solar tracking like stationary solar panels, MPPT etc have many disadvantages. Stationary mounted PV panels are only perpendicular to the sun once a day. In case of MPPT, the maximum power point obtained during shadow falling on panel have flaws and not reliable. So to overcome these disadvantages we proposed sensor based dual axis solar tracking system which rotates the panel in the direction of the sun where it gets maximum intensity of the sun light. Solar to Electrical energy conversion efficiency can be given by this formula:

$$
\eta=\frac{\boldsymbol{P}_{\boldsymbol{m}}}{\left(\boldsymbol{G} * \boldsymbol{A}_{\boldsymbol{c}}\right)}
$$

where: $P_{m}=$ maximum power in watt

$\mathrm{G}=$ irradiance (input light in watt $/ \mathrm{m}^{2}$ )

$\mathrm{A}_{\mathrm{c}}=$ surface area of solar cell in $\mathrm{m}^{2}$

Efficiency of a solar panel can by increasing $P_{m}$ (maximum power) of the panel. The proposed tracking system does tracking of sunlight more effectively by providing PV panel rotation in two different axes. In dual-axis tracking system optimum power is achieved by tracking the sun in four directions. In this way we can capture more sun rays. The dual-axis solar tracker follows the angular height position of the sun in the sky in addition to following the sun's east-west movement. The dual-axis working is similar to single axis but it captures the 
solar energy more effectively by rotating in the horizontal as well as the vertical axis. The tracker model is composed of four LDR sensors, two DC motors and Arduino microcontroller. One set of sensors and one motor is used to tilt the tracker in sun's east-west direction and the other set of sensors and the other motor which is fixed at the bottom of the tracker is used to tilt the tracker in the sun's north-south direction.

\subsection{Hardware Design}

This section deals with hardware used in model which includes study of panel, sensor selection and also their arrangement, motor selection and study of microcontroller.

\subsubsection{Schematics of Dual Axis Solar Tracker}

Model prototype consists of a 4 LDR arrangement which is housed on PV panel for detecting direction of maximum light intensity. Output of LDRs after proper signal conditioning is fed to Arduino microcontroller which drives the horizontal and vertical motor for dual axis tracking through tracking software to orient the solar panel in the direction of maximum sunlight.

\subsubsection{Hardware Components of Dual Axis Tracker Are:}

a. Sensor and sensing arrangement

b. Microcontroller

c. Actuators

d. Driver circuit.

The tracker model is composed of four LDR sensors in suitable sensing arrangement. One set of sensors and one motor is used to tilt the tracker in sun's east-west direction and the other set of sensors and the other motor which is fixed at the bottom of the tracker is used to tilt the tracker in the sun's north-south direction. DC motors are basically performing function of sun tracking. Upper panel holder DC motor tracks the sun linearly and base DC motor tracks the parabolic displacement of sun.

Specifications of the dc motor used:

a. Length: $80 \mathrm{~mm}$

b. Torque: $1.5 \mathrm{~kg} . \mathrm{cm}$

c. Shaft Diameter: $6 \mathrm{~mm}$

d. Weight: $130.00 \mathrm{~g}$

Microcontroller: These DC motors and sensors are interfaced with an Arduino microcontroller which is controlling DC motors on the basis of sensor's input. LDR sensors sense the light and sends signal to microcontroller. Microcontroller is doing comparison of signals received from LDR sensors and on the basis of stronger signal it is deciding rotation direction of DC motors. Dual Axis tracker control is explained with the help of block diagram Figure 1.

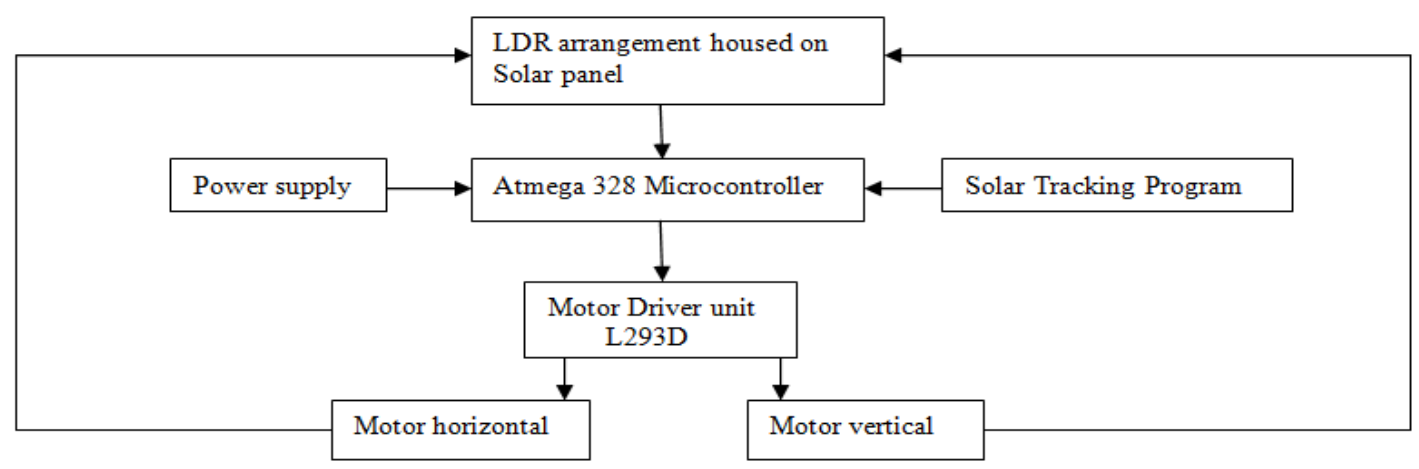

Figure 1. Block diagram of dual axis solar tracking system for solar panel

The block diagram is showing that LDR sensors after sensing the light forward the signal to Microcontroller. Microcontroller is intelligent device which is taking actions on the basis of 
sensor input and activating the motor driver's circuit (L293D) accordingly. Now suppose if sun changes it location and moves from east to west, it will cause light intensity to be different on one sensor as compared to other one. On the basis of light intensity difference on sensors, controller activates driver circuits and moves DC motors to new position where light falling on sensor is maximum.

\subsubsection{Light Sensing Arrangement}

This section deals with designing of a light intensity sensing arrangement such that at any point of time only one LDR detect maximum intensity of light. For this we have studied and selected Criss-Cross arrangement. In this arrangement we obstruct the light intensity falling on LDR in such a way that at any given point of time only one LDR will sense the maximum light. Figure 2 shows the front view and top view of criss-cross arrangement [8].

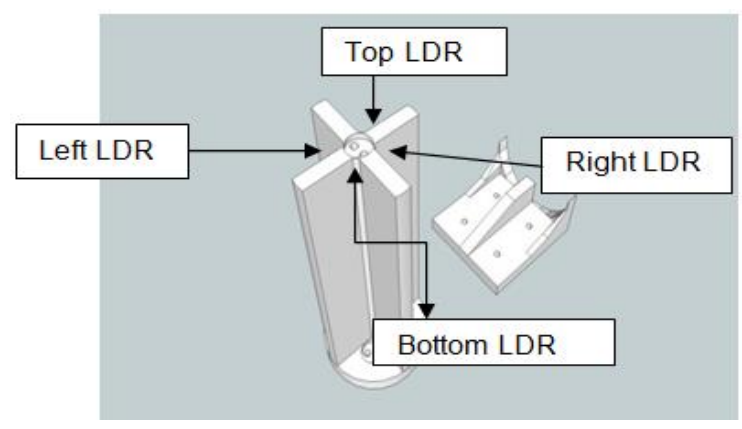

Figure 2. Light sensing arrangement (front view)

After signal conditioning of 4 LDR by connecting $10 \mathrm{k}$ ohm resistor in series with each LDR and voltage drop across each resistor is plotted. The LDR with maximum light intensity falling will have minimum resistance and voltage drop is also minimum across it therefore voltage drop across series resistor will be maximum. On basis of Figure 3, it can be seen that at any point of time values of all 4 voltage drop can be easily differentiated.

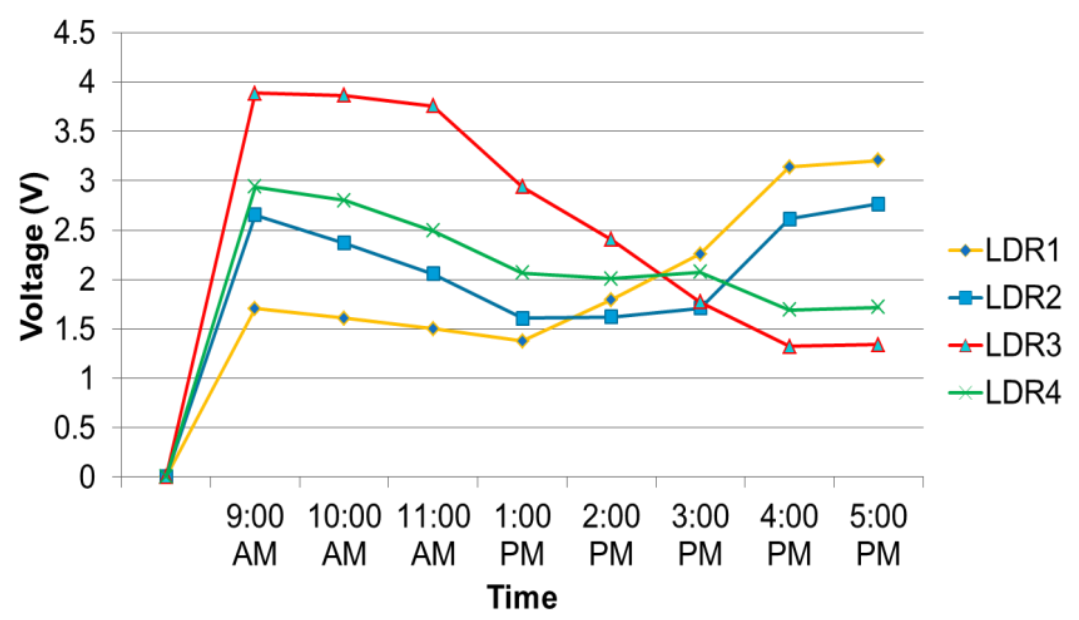

Figure 3. Voltage variation wrt time of 4 LDRs in criss cross arrangement

\subsubsection{L293D Motor Driver IC}

L293D is a typical motor driver IC which allows DC motor to drive on either direction. L293D is a 16 pin IC which can control a set of two DC motors simultaneously in any direction. It 
means that we can control two DC motor with single L293D IC. The L293D uses $5 \mathrm{~V}$ for its own power and external power source is needed to drive the motors. It works on the concept of $\mathrm{H}$ bridge. $\mathrm{H}$-bridge is the circuit which allows the voltage to be flown in either direction. There are 4 input pins for L293D, pin 2, 7 on the left and pin 15, 10 on the right as shown on the pin diagram. Left input pins will regulate the rotation of motor connected across left side and right input for motor on the right hand side. The motors are rotated on the basis of the inputs provided across the input pins as LOGIC 0 or LOGIC 1. Figure 4 shows L293D motor driver circuit.

L293D Logic Table:

Let's consider a Motor connected on left side output pins (pin 3,6). For rotating the motor in clockwise direction the input pins has to be provided with Logic 1 and Logic 0.

a. Pin 2=Logic 1 and Pin 7=Logic $0 \mid$ Clockwise Direction

b. Pin 2=Logic 0 and Pin 7=Logic $1 \mid$ Anticlockwise Direction

c. Pin 2=Logic 0 and Pin $7=$ Logic $0 \mid$ Idle [No rotation] [Hi-Impedance state]

d. Pin 2=Logic 1 and Pin 7=Logic 1 | Idle [No rotation]

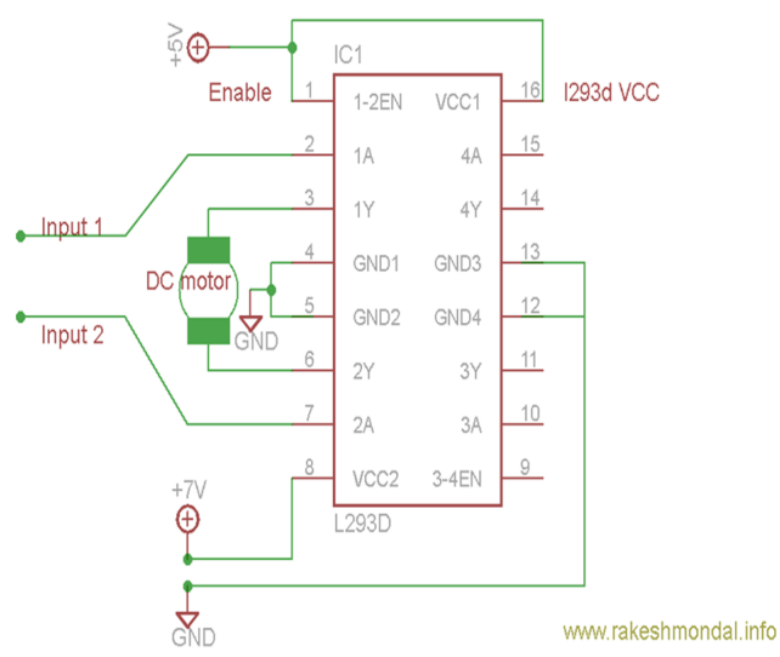

Figure 4. L293D motor driver circuit

\subsection{Solar Tracking System Software}

Tracking software designed can be categorized on the basis of functions into modules where each module performing unique function. These functional modules are given below-

\section{a. Data acquisition}

The very first requirement for software design is to acquire the sensor data. Sensor data here refers to the voltage reading of LDR which vary according to the light intensity of incident light. After signal conditioning of LDR, value of voltages is fed into analog port of arduino board for data acquisition.

\section{b. Maximum light intensity detection algorithm}

Detection of maximum intensity is based on data fusion where data fusion is the process of integration of multiple data and knowledge representing the same real-world object into a consistent, accurate, and useful representation. Light intensity falling on four LDRs in the same real world converted to voltages by connecting $10 \mathrm{kohm}$ resistor in series with each LDR and voltage drop across each resistor is monitored and maximum voltage across resistor represent maximum intensity of light falling on corresponding LDR.

\section{c. Sensing maximum light intensity direction}

By comparing the voltages we can detect which LDR has maximum light intensity on it. For example if the left LDR has maximum light intensity falling on it then resistor across it has maximum voltage drop so horizontal motor controlling the azimuth angle of panel is required to move clockwise [7]. 
Case 1: LDRL > LDRR, LDRB and LDRT

Motor controlling horizontal motion rotated clockwise by giving digital pin 7-LOW and pin 8HIGH to arduino pin which is fed as input to driver IC on pin 7 and pin 2 and drive motor clockwise.

Case 2: LDRT > LDRR, LDRB and LDRL

Motor controlling vertical motion rotated clockwise by giving digital pin 9-LOW and pin 10-HIGH of Arduino and it give input to pin 10 and 15 and drive vertical motor.

Case 3: LDRR > LDRL, LDRB and LDRT

Motor controlling horizontal motion rotated anti-clockwise by giving digital pin 7-HIGH and pin 8LOW to arduino pin which is fed as input to driver IC on pin 7 and pin 2 and drive motor anti clockwise.

Case 4: LDRB > LDRT, LDRR and LDRL

Motor controlling vertical motion rotated anti-clockwise by giving digital pin 9-HIGH and pin 10LOW and it give input to pin 10 and 15 and drive vertical motor.

d. Continuous mode of operation

For re-execution reset button is pressed and for continuous mode of operation timer switch is pressed which provide time span between two execution which is selected by user when both switch are in true state tracking algorithm runs again.

\section{Dual axis tracking algorithm}

It is initialize by declaring and initializing variables to take sensor values and feed it into arduino for comparision.SVR is sensor value for right LDR ,SVL for left ,SVT for top , SVB for bottom LDR and PV for panel voltage. Figure 5 shows dual axis tracking algorithm.

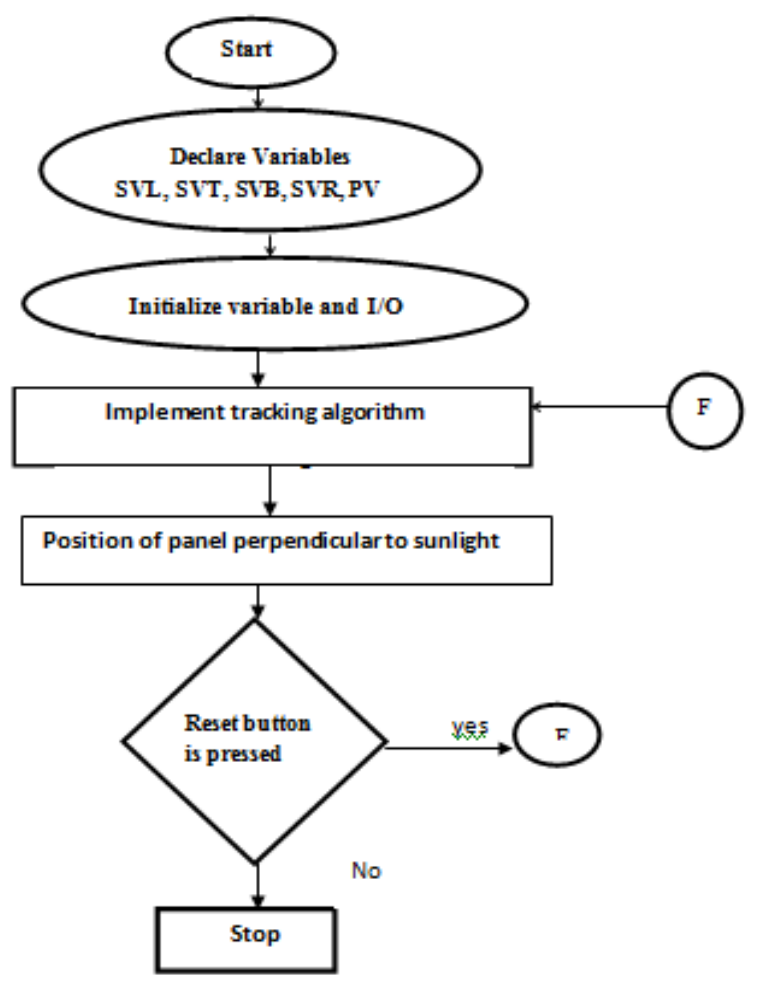

Figure 5. Dual axis tracking algorithm

\section{Model Prototype}

Figure 6 shows final prototype of dual axis solar tracking system for solar panel. 


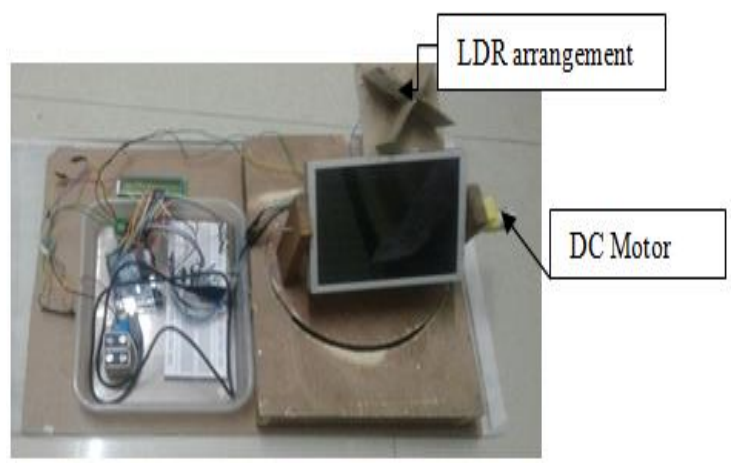

Figure 6. Final prototype of dual axis solar tracking system for solar panel

\section{Result}

After designing, fabrication and programming of our model the following results were obtained:

a. Laboratory Result.

b. 2 Outdoor Result.

c. Comparison Between Static and tracking system

1) Laboratory Result

In laboratory experiment the tracking testing has been conducted using torch. When the intensity of light is maximum on left LDR or right LDR, the horizontal motor rotates clockwise and anti clockwise respectively. Similarly when the light is maximum on top and bottom LDR, the vertical motor rotate accordingly.

2) Comparison Between Static and Tracking System

Table 1 shows static solar panel and tracking solar panel voltage variation with time. Where Figure 7 shows time vs voltage characteristics of static and tracking solar panel.

Table 1. Static Solar Panel and Tracking Solar Panel Voltage Variation with Time

\begin{tabular}{ccc}
\hline Time & $\begin{array}{c}\text { Static } \\
\text { Panel } \\
\text { Voltage(V) }\end{array}$ & $\begin{array}{c}\text { Solar } \\
\text { Tracking } \\
\text { Panel } \\
\text { Voltage }(V)\end{array}$ \\
\hline 10: $15 \mathrm{am}$ & 6.92 & 7.16 \\
11: $15 \mathrm{am}$ & 6.95 & 7.18 \\
12:15 pm & 7.17 & 7.25 \\
$01: 15 \mathrm{pm}$ & 7.18 & 7.20 \\
02:00 pm & 7.12 & 7.19 \\
02:30 pm & 7.02 & 7.17 \\
03: $00 \mathrm{pm}$ & 6.94 & 7.13 \\
$04: 00 \mathrm{pm}$ & 6.90 & 7.09 \\
$04: 45 \mathrm{pm}$ & 6.67 & 6.8 \\
\hline
\end{tabular}

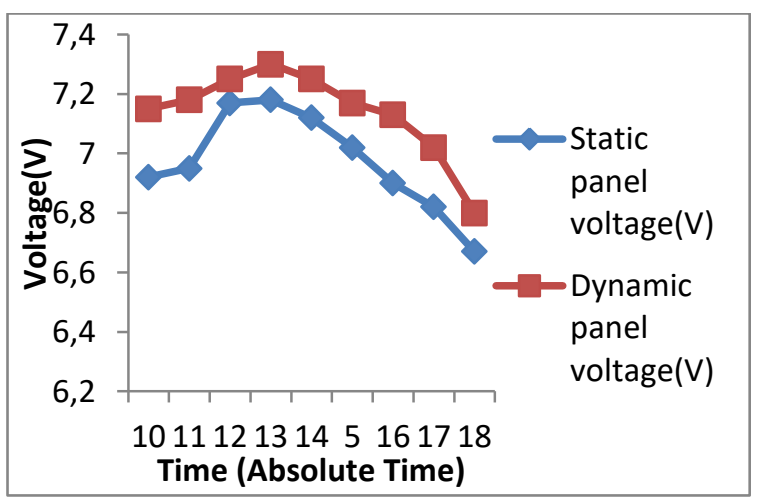

Figure 7. Time vs voltage characteristics of static and tracking solar panel 


\section{3) Outdoor Result}

This experiment was conducted in sunlight. In this the LDR which sense maximum sun light, will provide maximum value of voltage according to which the horizontal or vertical motor rotates. Figure 8 shows experiment was conducted in sunlight.
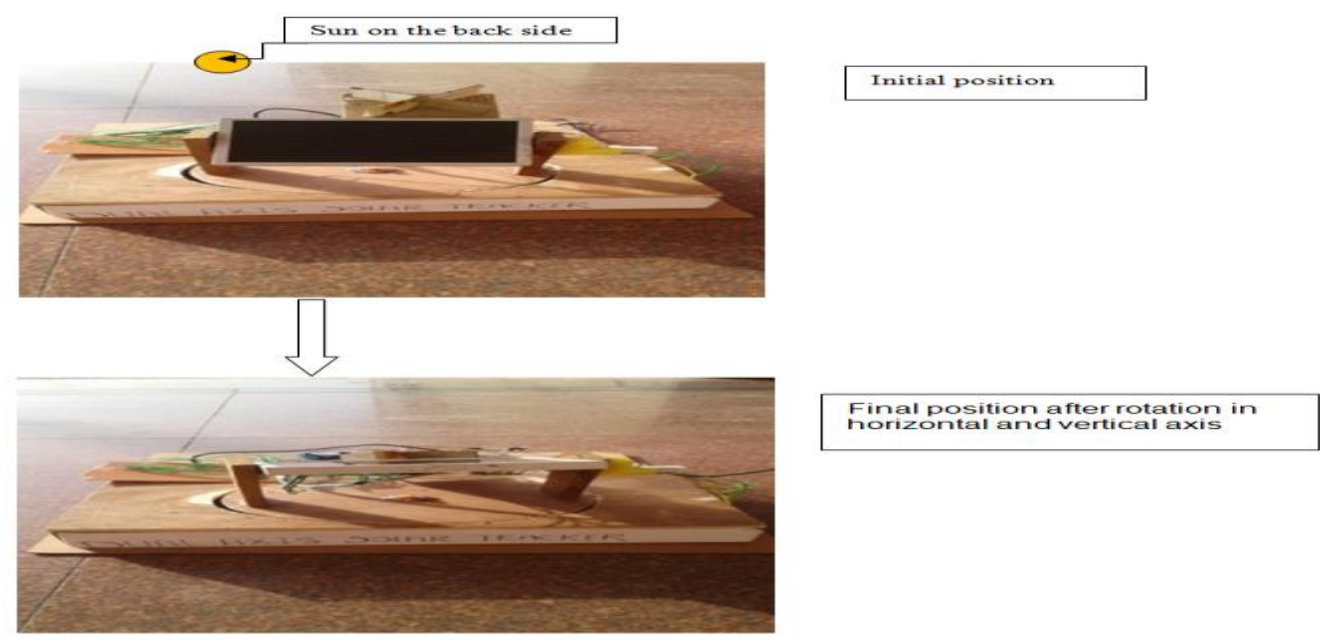

Figure 8. Experiment was conducted in sunlight

\section{Conclusion and Future Directions}

The prototype model for dual-axis sun tracking system for solar panel has been fabricated successfully. The working performance of the system during the day testing (on a full sunny day) with light intensity ranging from was found satisfactory. At each time increment of one hour, sensing arrangement detected the direction of maximum sunlight intensity and oriented the motor in the direction of sun. The output voltage of the solar panel with the tracking arrangement was found to be more than the static arrangement in which the direction of the panel remain same throughout the day approximately, there was a maximum increment of $3.5 \%$ in the output voltage was observed with the proposed dual axis tracking system. This will eventually provide the maximum power to the load at any given time instant. This project ensures that the percentage usage of available solar energy on solar panel has increased during the day, thus increasing its output voltage available to the load, thus fulfilling the basic aim of the work.It is a cost-effective re-programmable embedded system that can easily be upgraded to suit in future other tracking algorithms. The system developed can be successfully implemented to drive large or small solar panels both for commercial purposes as well as domestic applications.

\section{Future Directions}

a. Developments can be made regarding the design of the structure, for example by adding covers for the motors and also improving the design of the sensor holder by making it waterproof.

b. Improvements can be made to the current method of turning back the frame to its original position, removing the need to manually adjust the limit switches.

c. It can be designed as more user friendly system.

d. Dust particle detection and automatic cleaning system can also be incorporated in the system

\section{References}

[1] Chin CS, Babu A, Mc Bride. Design, Modeling and Testing of a Standalone Single Axis Active Solar Tracker UsingMATLAB/Simulink. Renewable Energy. 2011; 36: 3075-3090. 
[2] Prasad D, Snow. Designing with Solar Power: A Source Book for Building Integrated Photovoltaic (BiPV). London: Earthscan. 2005: 23.

[3] Goetz Berger A, Hebling C, Schock H. Photovoltaic Materials, History, Status and Outlook. Materials Science and Engineering: R: Reports. 2002; 40: 1-46.

[4] Mousazadeh H, Keyhani A, Javadi A, Mobli H, Abrinia K, Sharifi. A Review of Principle and SunTracking Methods. 2009.

[5] Binoy Seal, Omkar Shirke, Siddhesh Shewale, Abhilash Sirsikar. Solar Based Automatic Irrigation System. International Journal of Research in Advent Technology. 2014; 2(4):186.

[6] K Anusha, S Chandra Mohan Reddy. International Journal of Engineering Research and Applications (IJERA). 2013; 3(1): 1219-1223.

[7] Maximum power point technique a review. International Journal of Recent Research in Electrical and Electronics Engineering (IJRREEE). 2014; 1(1): 25-33.

[8] Nader Barsoum, Rinoe Nizam, Elvin Gerard. New Approach on Development a Dual Axis Solar Tracking Prototype. Wireless Engineering and Technology. Published Online January 2016 in SciRes. 2016; 7: 1-11. 